\title{
PATHO CLINICAL PROFILE OF DENGUE FEVER IN HOSPITALISED CHILDREN
}

\author{
Lakshmi Prasanna ${ }^{1}$, Gadham Jayaram *2.
}

${ }^{1}$ Assistant Professor in Pediatrics, Nimra Medical College, Jupudi, Andhra Pradesh, India.

${ }^{2}$ Associate Professor in Physiology, Nimra Medical College, Jupudi, Andhra Pradesh, India.

\section{ABSTRACT}

Dengue virus infection is recognized as one of the most important mosquito borne human infections in the $21^{\text {st }}$ century. Its incidence has increased in the present decade by 30 -fold with increasing geographic expansion to new countries, from urban to rural areas. Estimated 50million dengue infections occur annually and approximately 2.5 billion people live in dengue endemic countries.There has been increasing incidence of dengue fever in this part in recent years. Dengue fever is known to involve multiple systems sometimes resulting multi organ dysfunction. Bleeding manifestation is most common to occur in dengue fever. Hence the following prospective observational study in 500 consecutive patients with dengue fever was study was conducted at Nimra Medical College in 2 years (November 2015 to November 2017) to find out to know the correlation between the clinical bleeding and laboratory parameters which significantly correlate to clinical bleeding in patients with DF, DHF, and DSS.Out of 500 children, $61 \%$ belongs to DF among which $98.36 \%$ were recovered, $1.63 \%$ were expired, $23 \%$ belongs to DHF and are $100 \%$ recovered, and $16 \%$ DSS of which $97.50 \%$ were recovered, $2.50 \%$ were expired. Bleeding manifestations are very common in dengue infection with altered laboratory parameters. Thus, bleeding manifestations and low platelet count with altered coagulation profile in serologically positive cases can be used to guard the prognosis of dengue fever and DHF.

KEY WORDS: Dengue Fever, Serology, Hb\%, PCV, TLC, DLC, Platelet Count, BT, and CT.

Address for correspondence: Dr. Gadham Jayaram, Associate Professor in Physiology, Nimra Medical College, Jupudi, Andhra Pradesh, India. E-Mail: drjayaram@yahoo.com

\begin{tabular}{|l|l|}
\hline \multicolumn{3}{|c|}{ Online Access and Article Informtaion } \\
\hline Quick Response code & \multicolumn{1}{|c|}{ International Journal of Integrative Medical Sciences } \\
& \multicolumn{1}{c|}{ ISSN (P): 2394 - 6318. ISSN (E): 2394 - 4137 } \\
www.imedsciences.com
\end{tabular}

\section{BACKGROUND}

Dengue (West Nile fever) is currently regarded globally as the most important mosquito-borne viral disease, which causes fever, rashes and muscle and joint aches. Dengue fever cased by the arbovirus such as West Nile virus, endemoepidemic arboviral infection in many tropical and subtropical regions of the world. In the past 50 years, the prevalence of dengue fever has increased 30 fold with increasing geographic expansion to new countries and in the present decade, from urban to rural setting. A history of symptoms compatible with dengue can be traced back to the Chin Dynasty of 265-420 AD [1]. The virus is winning distributed throughout tropical and subtropical regions of the world, particularly over the last half-century Significant geographic expansion has been coupled with rapid increases in incident cases, epidemics, and hyperendemicity, leading to the more severe forms of dengue. Transmission of dengue is now present in every World Health Organization (WHO) region of the world and more than 125 countries are known to be dengue endemic [1].

2.5 to 3 billion individual's global population at risk living mainly in urban areas in tropical and subtropical regions. There are at least100 million cases of dengue fever annually and 500,000 
cases of DHF which require hospitalization. Of the latter, $90 \%$ are children under the age of 15 years. DHF mortality rates average $5 \%$, with approximately 25,000 deaths each year Mortality averages $5 \%$ of DHF cases. Case fatality rates vary from 1 to $5 \%$ but can be $<1 \%$ with appropriate treatment [2]. Hemorrhagic manifestations in dengue infection seen clinically are petechiae, echymosis, purpurae, hematuria, hemoptysis, subconjunctival hemorrhages, epistaxis, hematemesis, skin bleeds, malena, gum bleeds, menorrhagia, positive tourniquet test are seen clinically $[3,4]$, and laboratory parameters which are seen in dengue fever and dengue hemorrhagic fever are platelet count $<1,00,000 /$ cumm, hematocrit $>40 \%$, abnormal coagulation profile, leucopenia / leukocytosis [3,4]. Bleeding in dengue fever is multifactorial in etiology. Several factors like, thrombocytopenia, prolonged shock with capillary leak, derangement of coagulation profile, are known causes of bleeding. Since our hospital (Nimra Medical College, Jupudi, A.P) are tertiary care hospital, we do see a lot of children with dengue infection including children with bleeding manifestation. So an attempt has been made to know the correlation between the clinical bleeding and laboratory parameters.

\section{MATERIALS AND METHODS}

The present study was conducted at Nimra Medical College, Jupudi, A.P. in 1 years (November 2015 to November 2017). There has been increasing incidence of dengue fever in this part in recent years. Dengue fever is known to involve multiple systems sometimes resulting multi organ dysfunction. Bleeding manifestation is most common to occur in dengue fever. Hence the following prospective observational study in 500 consecutive patients with dengue fever was conducted to find out the bleeding risk in children with dengue viral infection. So an attempt has been made to know the correlation between the clinical bleeding and laboratory parameters which significantly correlate to clinical bleeding in patients with DF, DHF, and DSS. Patients were having signs and symptoms of dengue fever and who also had aged between new born to 17 years and positive for dengue IgM, IgG AND NS1 and all IgM/IgG/NS1 antibodies in the blood were included in the study.
Children aged $>17$ years of age, having IgM and IgG negative and develop hemorrhagic manifestation unrelated to dengue fever and refuse to be include in the study was excluded.All the children in the study will be investigated for Dengue Serology, Hemoparasites, Peripheral Blood Smear, Abdominal Ultra Sound, Hb\%, PCV, TLC, DLC, Platelet Count, BT, CT.

\section{RESULTS}

Table 1: Age Distribution.

\begin{tabular}{|c|c|c|c|c|}
\hline Age (years) & No. of cases & DF & DHF & DSS \\
\hline 1-4 Years & 185 & 117 & 34 & 34 \\
\hline $\mathbf{4 . 1 - 8}$ Years & 174 & 106 & 42 & 26 \\
\hline $\mathbf{8 . 1 - 1 2}$ Years & 91 & 53 & 28 & 10 \\
\hline $\mathbf{1 2 . 1 - 1 6}$ years & 46 & 27 & 9 & 10 \\
\hline $\mathbf{1 6 . 1 - 1 8}$ years & 4 & 2 & 2 & 0 \\
\hline Total & 500 & 305 & 115 & 80 \\
\hline
\end{tabular}

Table 2: Comparison of serologically positive cases.

\begin{tabular}{|c|c|c|c|c|c|}
\hline IgM/IgG & Total & D F & DHF & DSS & P* Value \\
\hline IgM & 45 & 30 & 11 & 4 & \\
\cline { 1 - 5 } IgG & 52 & 28 & 14 & 10 & \multirow{2}{*}{0.54} \\
\cline { 1 - 5 } NS1 & 59 & 31 & 16 & 12 & \\
\cline { 1 - 5 } IgM/IgG/NS1 & 1 & 1 & 0 & 0 & \\
\hline
\end{tabular}

Table 3: Clinical spectrum of cases at time of admission.

\begin{tabular}{|c|c|c|c|c|c|}
\hline \multirow{2}{*}{ Group } & \multirow{2}{*}{$\begin{array}{c}\text { No. of } \\
\text { cases }\end{array}$} & \multicolumn{2}{|c|}{ Recovered } & \multicolumn{2}{c|}{ Expired } \\
\cline { 3 - 6 } & & $\begin{array}{c}\text { No. Of } \\
\text { cases }\end{array}$ & $\%$ & $\begin{array}{c}\text { No. Of } \\
\text { cases }\end{array}$ & $\%$ \\
\hline DF & 305 & 300 & $98.36 \%$ & 5 & $1.63 \%$ \\
\hline DHF & 115 & 115 & $100 \%$ & 0 & $0 \%$ \\
\hline DSS & 80 & 78 & $97.50 \%$ & 2 & $2.50 \%$ \\
\hline Total & 500 & 493 & $98.60 \%$ & 7 & $1.40 \%$ \\
\hline
\end{tabular}

Table 4: Relationship between various sites of bleeding at the time of admission.

\begin{tabular}{|c|c|c|c|c|c|}
\hline Clinical Features & $\begin{array}{c}\text { Total } \\
(\mathrm{n}=\mathbf{5 0 0})\end{array}$ & $\begin{array}{c}\text { DF } \\
(\mathrm{n}=\mathbf{3 0 5})\end{array}$ & $\begin{array}{c}\text { DHF } \\
(\mathrm{n}=\mathbf{1 1 5})\end{array}$ & $\begin{array}{c}\text { DSS } \\
(\mathrm{n}=\mathbf{8 0})\end{array}$ & P* Value \\
\hline PE/PU/ EC & 57 & 28 & 14 & 15 & 0.02 \\
\hline Malena & 198 & 99 & 56 & 43 & $<0.001$ \\
\hline Epistaxis & 52 & 25 & 17 & 10 & $<0.001$ \\
\hline Gum bleeds & 35 & 16 & 11 & 8 & 0.005 \\
\hline Menorrhagia & 3 & 1 & 0 & 2 & 0.07 \\
\hline Eye hemorrhage & 78 & 41 & 17 & 20 & $<0.001$ \\
\hline Hematuria & 12 & 5 & 3 & 4 & 0.18 \\
\hline Hematemesis & 0 & 0 & 0 & 0 & - \\
\hline
\end{tabular}


Table 5: Haemoglobin Level, Total Leukocyte Count, Platelet Counts at Admission.

\begin{tabular}{|c|c|c|c|c|c|}
\hline & & $\begin{array}{c}\text { Total } \\
(n=500)\end{array}$ & $\begin{array}{c}\text { DF } \\
(n=305)\end{array}$ & $\begin{array}{c}\text { DHF } \\
(n=115)\end{array}$ & $\begin{array}{c}\text { DSS } \\
(n=80)\end{array}$ \\
\hline \multirow{3}{*}{ HB\% } & Upto8.0 & 51 & 34 & 10 & 7 \\
\hline & $8.0-12.0$ & 226 & 138 & 52 & 36 \\
\hline & $>12.0$ & 223 & 133 & 53 & 37 \\
\hline \multirow{3}{*}{ TLC } & $<4000$ & 98 & 65 & 75 & 50 \\
\hline & $4000-11000$ & 342 & 204 & 27 & 19 \\
\hline & $>11000$ & 60 & 36 & 13 & 11 \\
\hline \multirow{6}{*}{ Platelet } & $<20,000$ & 24 & 16 & 5 & 3 \\
\hline & $20,001-50,000$ & 98 & 56 & 30 & 12 \\
\hline & $50,001-75,000$ & 71 & 39 & 21 & 11 \\
\hline & $75,001-100,000$ & 22 & 10 & 6 & 6 \\
\hline & $100,01-150,000$ & 103 & 67 & 16 & 20 \\
\hline & $>150,000$ & 182 & 117 & 37 & 28 \\
\hline
\end{tabular}

Table 6: Comparision of Haemoglobin Level, Total Leukocyte Count, Platelet Counts, BT (Sec), and Clotting

Time in dengue patients.

\begin{tabular}{|c|c|c|c|c|c|c|}
\hline \multicolumn{2}{|c|}{} & $\begin{array}{c}\text { Total } \\
(\mathbf{n = 5 0 0})\end{array}$ & $\begin{array}{c}\text { D F } \\
(\mathbf{n = 3 0 5})\end{array}$ & $\begin{array}{c}\text { DHF } \\
(\mathbf{n = 1 1 5})\end{array}$ & $\begin{array}{c}\text { DSS } \\
(\mathbf{n = 8 0})\end{array}$ & P* Value \\
\hline \multirow{2}{*}{ HB\% } & Mean & 11.26 & 11.13 & 11.41 & 11.55 & \multirow{2}{*}{0.001} \\
\cline { 2 - 7 } & SD & 2.66 & 2.64 & 2.76 & 2.56 & \\
\hline \multirow{2}{*}{ TLC } & Mean & 7136.61 & 7117.78 & 7078.84 & 7291.45 & \multirow{2}{*}{0.79} \\
\cline { 2 - 7 } & SD & 2894.29 & 2916.55 & 2799.93 & 2972.57 & \\
\hline \multirow{2}{*}{ Platelet } & Mean & 149588 & 157521.31 & 137304.35 & 137000 & \multirow{2}{*}{0.001} \\
\cline { 2 - 7 } & SD & 140054.4 & 148518.7 & 137262.73 & 102861.15 & \\
\hline \multirow{2}{*}{ BT (Sec) } & MEAN & 283.57 & 282.34 & 288.15 & 281.65 & \multirow{2}{*}{0.003} \\
\cline { 2 - 7 } & SD & 61.82 & 63.3 & 61.72 & 56.38 & \multirow{2}{*}{0.35} \\
\hline \multirow{2}{*}{ Clotting } & MEAN & 345.19 & 344.13 & 345.17 & 349.25 & \\
\cline { 2 - 7 } Time & SD & 44.21 & 44.78 & 44.01 & 42.58 & \\
\hline
\end{tabular}

\section{DISCUSSION}

Dengue is most frequent viral infection that affects a wide range of population globally and particularly pediatric population. Epidemiological studies have shown that dengue fever affects more than 100 million populations annually throughout the world with higher prevalence of morbidity manifested with complications including dengue hemorrhagic fever and dengue shock syndrome and higher incidence of mortality. It is hence prompting clinicians for early diagnosis of dengue by integrated approach of bed side clinical examination, supported and confirmed by laboratory and radiological diagnosis, prevention of dreadful complications and comprehensive management including the associated complications. Most of the studies have studied on the varied clinical presentation of dengue fever that range from non-specific febrile illness to dengue hemorrhagic fever or DSS. It is the reticulo endothelial system that is affected due virus infection leading to aberrant endothelial damage of vascular bed and finally presenting as bleeding manifestations from various sites. However, very few studies have showed the clinical and laboratory profile in pediatric population with reference to South Indian population and diagnostic importance of NS1, IgM and IgG antibodies testing in early detection of dengue fever and need for prompt management to prevent complications.

In the present study, it was observed that mean age at the time of presentation of child with dengue fever was $8.4 \pm 1.2$ years. This is in agreement with earlier studies by Chandrakanta et al (5), Alam et al (6), Prakash O. et al (7). It was observed that 4-12 year age group children dominated in the present study accounting to $53 \%$ followed by $0-4$ years age group. Lower incidence of dengue fever is observed in age group $>12$ years. Among the various types of presentation of dengue fever with or without 
complications as DHF or DSS, the present study has showed that higher incidences of dengue fever was observed with earlier age groups when compared to increasing age of the children. However no significant difference was observed in relation to age group and dengue fever with associated complications.

It was observed from the present study that male predominance with $\mathrm{M}: \mathrm{F}=1.4: 1$ was observed in overall group and also the predominance of male children across all the subgroups and are in accordance to previous studied by WHO 1999 [8], Wang CC et al [9], Ahmed MM et al [10], Josi R. et al [11], Chandrakanta et al [5], Shah I. et al [12].

The most serious complications associated with dengue fever and mortality includes DHF and DSS are serious clinical manifestation of the dengue infection. The relative incidence of children presenting with DF, DHF, DSS in the present study was observed to be $61 \%, 23 \%$ and $16 \%$. However, the incidence of DHF in the present study was observed slightly higher when compared to earlier studied by Joshi et al [11] and Nag CF et al [13].

In the present study 500 children in the study all children had fever as symptom at the time of admission. the most important primary symptom at the presentation in patients with dengue fever was fever with $100 \%$ followed by vomiting $67 \%$, abdominal pain $36 \%$, bleeding manifestations $43 \%$, and the least incidence of clinical presentation in dengue fever observed was periorbital pain $23 \%$. The results of the present study have been comparable and are agreement with earlier studies by Naryanan et al [14] and Joshi R. et al [11].

The mean hemoglobin and hematocrit in the present study were $11.2 \mathrm{gm} / \mathrm{dl}$ and $35.2 \%$ respectively. There was a significant statistical correlation between hematocrit and severity of disease among the clinical subgroups of dengue. Naryan et al reported the same to be $10.8 \mathrm{gm} \%$ and $33.2 \%$ respectively [14].

The classical description of $>\mathbf{2 0 \%}$ rise in the hematocrit is difficult to establish, as the reference standards have not been established for children. Hence the rise in hematocrit was taken one of the diagnostic criteria in identifying dengue fever. Gomber et al had defined a cut off haematocrit value as $36.3 \%$ to be diagnostic of DHF in Indian population [15]. Accounting of this value, children in present study came under the category of DHF, which correlated with clinical staging. Although leucopenia has been reported in large number of studies, the present study had a mean total leukocyte count of 7136 cells/cumm. The highest and lowest TLC was 14523 and 1253 /cumm respectively. One of the most important diagnostic clue in the diagnosis of dengue fever is abnormal low platelet count. On taking the WHO limit of $<100000 /$ cumm for low platelet count, $43 \%$ of children had in the present study. The mean platelet in the present study was $145,860 /$ cumm with range of 12000 , to $425,000 /$ cumm. Only platelet count at admission was not taken as an indicator for bleeding tendencies. These suggest that other factors like platelet dysfunction or disseminated intravascular coagulation may have a role in bleeding in dengue fever cases. However studies which include only DHF cases show correlation between low platelet count and bleeding manifestation [16]. The studies by Gomber et al and Narayan et al have documented the same $[14,15]$. Platelet count provides a very useful means of diagnoses at the screening level. Hence platelet count was a sensitive indicator for diagnosis. Bleeding manifestations are more frequent with low platelet count.

In the present study bleeding manifestation were found in $43 \%$. The incidence of bleeding in our study was lower when compared to Anuradha $S$ et al [17] (52.6\%) and Alam ABMS et al (18) (54.3\%) studies. Malena was the most common bleeding manifestation in our study. We observed that bleeding time was increased in DSS and it was significant statistically $(p=0.003)$. Bleeding time can be used as a bed side tool to predict liver involvement or severe disease. There was no significant change in clotting time in the present study. The dengue IgM was tested positive in $29 \%$ of cases, IgG was tested positive in $30.4 \%$ and NS1 positive in $39.2 \%$ which show the importance of NS1 as an early antigen that can be useful in detection of dengue fever cases. However, combined use of all the antibodies in diagnosing dengue has been positive in $1 \%$ cases. Serology with clinical 
presentation and other investigations formed the mainstay of diagnosis. In the present study, it was observed that $79 \%$ of patients diagnosed with serologically positive dengue fever had hepatomegaly, followed by $76.3 \%$ with Pericholecystic edema, $76.1 \%$ with Gallbladder wall thickening, and $68.5 \%$ with as cites, $51 \%$ with Rt Pleural effusion and $39.2 \%$ with Splenomegaly. It is also observed that there significant association between all the mentioned Ultrasonographic findings with various types of serologically diagnosed dengue suggesting that ultrasonography may be helpful in guiding to diagnosis of dengue fever.

\section{CONCLUSION}

Out of 500 children, $61 \%$ belongs to DF among which $98.36 \%$ were recovered, $1.63 \%$ were expired, $23 \%$ belongs to DHF and are $100 \%$ recovered, and $16 \%$ DSS of which $97.50 \%$ were recovered, $2.50 \%$ were expired. In conclusion, it can be conveyed that Platelet count at admission alone is not sufficient to predict the possibility of bleeding in Dengue Fever. Also, Complete Coagulation Profile including BT, CT, PT and a PTT will also be required to predict severe hemorrhagic manifestations so as to have a better preparedness.

\section{REFERENCES}

[1]. Murray NE, Quam MB, Wilder-Smith A. Epidemiology of dengue: past, present and future prospects. Clin Epidemiol. 2013:20;5:299-309.

[2]. WHO. Prevention and control of dengue and dengue hemorrhagic fever 2013 New Delhi. World Health Organization, 2013.

[3]. Anuradha S, Singh NP, Rizvi SN, Agarwal SK, Gur R, Mathur MD. The 1996 outbreak of dengue hemorrhagic fever in Delhi, India. Southeast Asian J Trop Med Public Health. 1998;29(3):503-6.

[4]. Kabra SK, Jain Y, Singhal T, Ratageri VH. Dengue hemorrhagic fever: clinical manifestations and management. Indian J Pediatr. 1999;66(1):93-100.

[5]. chandrakanta, Kumar R, Garima, Agarwal J, Jain A, Nagar R.changing clinical manifestation of dengue infection in north India. dengue bulletin 2008;vol 132.
[6]. Alam AS, Sadat SA, Swapan Z, Ahmed AU, Karim MN, Paul HK, et al. Clinical profile of dengue fever in children. Bangladesh J Child Health. 2009;33(2):558.

[7]. Parkash O, Almas A, Jafri SM, Hamid S, Akhtar J, Alishah $\mathrm{H}$. Severity of acute hepatitis and its outcome in patients with dengue fever in a tertiary care hospital Karachi, Pakistan (South Asia). BMC Gastroenterology. 2010; 10(43):1-8.

[8]. World Health Organization. Prevention and Control of Dengue and Dengue Hemorrhagic Fever. WHO SEARO Publication No.29, New Delhi 1999.

[9]. Wang CC, Lee IK, Su MC, Lin HI, Huang YC, Liu SF, Wu CC, Lin MC. Differences in clinical and laboratory characteristics and disease severity between children and adults with dengue virus infection in Taiwan, 2002.Trans $R$ Soc Trop Med Hyg. 2009;103(9):871-7.

[10]. Ahmed MM. Clinical profile of dengue fever infection in King Abdul Aziz University Hospital Saudi Arabia. J Infect DevCtries. 2010 3;4(8):503-10

[11]. Joshi R, Baid V. Profile of dengue patients admitted to a tertiary care hospital in Mumbai. Turk J Pediatr. 2011;53(6):626-31.

[12]. Shah I, Katira B. Clinical and laboratory profile of dengue, leptospirosis and malaria in children: a study from Mumbai. Arch Dis Child. 2007;92(6):561.

[13]. Ng CF, Lum LC, Ismail NA, Tan LH, Tan CP. Clinicians' diagnostic practice of dengue infections. J Clin Virol. 2007;40(3):202-6.

[14]. Narayanan M, Aravind MA, Thilothammal N, Prema $R$, SargunamCS, Ramamurty N. Dengue fever epidemic in Chennai - a study of clinical profile and outcome. Indian Pediatr. 2002;39(11):1027-33.

[15]. Gomber S, Ramachandran VG, Kumar S, Agarwal KN, Gupta P, GuptaP, Dewan DK. Hematological observations as diagnostic markers in dengue hemorrhagic fever-a reappraisal. Indian Pediatr. 2001;38(5):477-81

[16]. Aggarwal A, Chandra J, Aneja S, Patwari AK, Dutta AK. An epidemic of dengue hemorrhagic fever and dengue shock syndrome in children in Delhi. Indian Pediatr. 1998 35(8):727- 32.

[17]. Anuradha S, Singh NP, Rizvi SN, Agarwal SK, Gur R, Mathur MD. The 1996 outbreak of dengue hemorrhagic fever in Delhi, India. Southeast Asian J Trop Med Public Health. 1998;29(3):503-6.

[18]. Alam ABMS, Sadat SA, Swapan Z, Ahmed AU, Karim MdN, Paul HK, Zaman S. Clinical Profile of Dengue Fever in Children. Bangladesh J Child Health. 2009;33(2):55-8.

How to cite this article: Lakshmi Prasanna, Gadham Jayaram. PATHO CLINICAL PROFILE OF DENGUE FEVER IN HOSPITALISED CHILDREN. Int J Intg Med Sci 2018;5(7):700-704. DOI: 10.16965/ijims.2018.126 